\title{
Erratum to: Association mapping of North American spring wheat breeding germplasm reveals loci conferring resistance to Ug99 and other African stem rust races
}

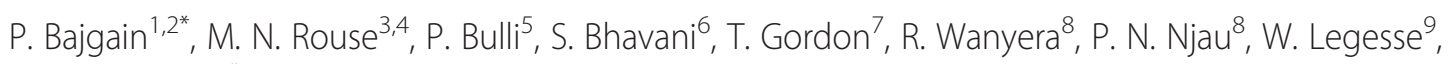
J. A. Anderson ${ }^{2^{*}}$ and M. O. Pumphrey ${ }^{5}$

\section{Erratum}

Upon publication of this article [1] it was noticed that Tables Two-Seven (Tables 1, 2, 3, 4, 5 and 6 here) were formatted incorrectly. The Tables have now been updated in the original article and can also be seen below.

\footnotetext{
*Correspondence: bajga002@umn.edu; ander319@umn.edu
}

1 Department of Agronomy, Purdue University, 915 West State Street, West Lafayette 47907, IN, USA

${ }^{2}$ Department of Agronomy and Plant Genetics, University of Minnesota, St. Paul 55108, MN, USA

Full list of author information is available at the end of the article 
Table 1 List of SNPS significantly associated with APR to the Ug99 race group in Kenya and Ethiopia. 'Chromosome 0' includes all unmapped SNP markers

\begin{tabular}{|c|c|c|c|c|c|c|c|c|}
\hline SNP & $\mathrm{Chr}^{\mathrm{b}}$ & Pos (cM) & $P$ value & $R^{2}(\%)^{c}$ & KenOff1 $3^{d}$ & KenMain13 & EthOff13 ${ }^{f}$ & EthOff1 $14^{g}$ \\
\hline IWB1351 & 0 & 0.0 & $9.18 \mathrm{E}-04$ & 1.4 & - & - & - & + \\
\hline IWB1 1987 & 0 & 0.0 & 2.71E-04 & 4.0 & - & - & - & - \\
\hline IWB13304 & 0 & 0.0 & $9.18 \mathrm{E}-04$ & 1.4 & - & - & - & + \\
\hline IWB20617 & 0 & 0.0 & 8.92E-04 & 0.5 & - & - & - & - \\
\hline IWB40153 & 0 & 0.0 & $9.18 \mathrm{E}-04$ & 1.4 & - & - & - & + \\
\hline IWB65634 & 0 & 0.0 & $2.18 \mathrm{E}-04$ & 3.9 & - & - & - & - \\
\hline IWA3120 & $1 \mathrm{~B}$ & 90.3 & $9.07 E-04$ & 0.6 & - & - & - & - \\
\hline IWB21176 & $1 \mathrm{~B}$ & 90.3 & 9.64E-04 & 0.6 & - & - & + & - \\
\hline IWB31027 & $1 \mathrm{~B}$ & 90.3 & $9.64 \mathrm{E}-04$ & 0.6 & - & - & + & - \\
\hline IWB56771 & $1 \mathrm{~B}$ & 90.3 & $9.34 \mathrm{E}-04$ & 0.6 & - & - & + & - \\
\hline IWB59663 & $1 \mathrm{~B}$ & 90.3 & $9.64 \mathrm{E}-04$ & 0.6 & - & - & + & - \\
\hline IWB49915 & $2 \mathrm{~A}$ & 122.5 & $9.18 \mathrm{E}-04$ & 1.4 & - & - & - & + \\
\hline IWB49914 & $2 \mathrm{~A}$ & 123.6 & $9.18 \mathrm{E}-04$ & 1.4 & - & - & - & + \\
\hline IWB22672 & $2 \mathrm{~A}$ & 159.7 & $9.98 \mathrm{E}-04$ & 0.6 & - & - & + & - \\
\hline IWB2369 & $2 B$ & 48.5 & $7.68 \mathrm{E}-04$ & 0.4 & - & - & - & - \\
\hline IWA4275 & $2 \mathrm{~B}$ & 105.9 & 8.75E-04 & 1.4 & - & - & - & - \\
\hline IWA8534 & $2 B$ & 126.1 & $1.80 \mathrm{E}-04$ & 0.3 & - & + & - & - \\
\hline IWB23660 & $2 \mathrm{~B}$ & 126.3 & $1.79 \mathrm{E}-04$ & 0.3 & - & + & - & - \\
\hline IWB25868 & $2 B$ & 126.3 & $3.11 \mathrm{E}-04$ & 0.7 & - & + & - & - \\
\hline IWB69631 & $2 \mathrm{~B}$ & 126.3 & $3.11 \mathrm{E}-04$ & 0.7 & - & + & - & - \\
\hline IWB25869 & $2 B$ & 126.5 & $3.11 \mathrm{E}-04$ & 0.7 & - & + & - & - \\
\hline IWB32143 & $2 \mathrm{~B}$ & 157.2 & 5.05E-04 & 4.6 & - & - & - & - \\
\hline IWB8481 & $2 \mathrm{D}$ & 9.2 & 8.44E-04 & 0.5 & - & - & - & - \\
\hline IWA5203 & $3 B$ & 11.5 & 7.97E-04 & 0.4 & - & - & - & - \\
\hline IWB30730 & $3 B$ & 11.5 & 7.74E-04 & 1.3 & - & - & - & - \\
\hline IWB12193 & $3 B$ & 11.6 & $3.24 \mathrm{E}-04$ & 4.2 & - & + & - & - \\
\hline IWB49924 & $3 B$ & 11.6 & 5.65E-04 & 0.2 & - & - & - & - \\
\hline IWB65737 & $3 B$ & 11.6 & $9.15 E-04$ & 0.6 & - & - & - & - \\
\hline IWB60424 & $3 B$ & 13.8 & $5.02 \mathrm{E}-04$ & 0.1 & - & - & - & - \\
\hline IWB36021 & $3 B$ & 14.1 & $8.31 \mathrm{E}-04$ & 1.3 & - & - & - & - \\
\hline IWA2493 & $3 B$ & 32.2 & 1.49E-04 & 0.2 & - & - & + & - \\
\hline IWB40004 & $4 \mathrm{~A}$ & 30.9 & $9.12 \mathrm{E}-04$ & 0.6 & - & + & - & - \\
\hline IWB52694 & $4 \mathrm{~A}$ & 43.4 & 9.96E-05 & 3.2 & - & + & - & - \\
\hline IWB46973 & $4 \mathrm{~A}$ & 47.0 & $8.26 \mathrm{E}-04$ & 1.3 & - & + & - & - \\
\hline IWB56556 & $4 \mathrm{~A}$ & 47.0 & $7.54 \mathrm{E}-04$ & 1.3 & - & - & - & - \\
\hline IWB67877 & $4 \mathrm{~A}$ & 47.0 & 8.34E-04 & 1.3 & - & - & - & - \\
\hline IWB47184 & $5 \mathrm{~A}$ & 69.6 & 3.44E-04 & 0.3 & - & - & + & - \\
\hline IWA233 & $6 \mathrm{~A}$ & 66.0 & $8.48 \mathrm{E}-04$ & 1.3 & - & + & - & - \\
\hline IWB24757 & $6 B$ & 119.7 & $6.33 \mathrm{E}-04$ & 1.1 & - & - & - & - \\
\hline IWB35697 & $6 \mathrm{~B}$ & 119.7 & 1.77E-05 & 1.3 & - & - & + & + \\
\hline IWB6474 & $6 \mathrm{~B}$ & 119.7 & $6.33 \mathrm{E}-04$ & 1.1 & - & - & - & - \\
\hline IWB45581 & $6 \mathrm{~B}$ & 120.6 & $3.00 \mathrm{E}-04$ & 0.6 & - & - & - & - \\
\hline IWB5070 & $7 \mathrm{~A}$ & 211.0 & 2.19E-04 & 3.9 & - & - & - & - \\
\hline
\end{tabular}


Table 1 List of SNPs significantly associated with APR to the Ug99 race group in Kenya and Ethiopia. 'Chromosome 0' includes all unmapped SNP markers (Continued)

\begin{tabular}{llllllll}
\hline IWB1874 & 7A & 212.7 & $3.18 \mathrm{E}-04$ & 0.3 & - & - & - \\
IWB4830 & $7 \mathrm{~A}$ & 212.7 & $4.50 \mathrm{E}-04$ & 4.5 & - & - & - \\
IWB62560 & 7A & 213.2 & $2.30 \mathrm{E}-04$ & 0.6 & - & - & - \\
IWB47548 & $7 \mathrm{~B}$ & 153.4 & $6.50 \mathrm{E}-04$ & 0.3 & - & - & - \\
IWA4175 & $7 \mathrm{~B}$ & 177.1 & $9.52 \mathrm{E}-04$ & 0.6 & - & - & - \\
\hline
\end{tabular}

aUnderlined SNP markers were detected during the 'combined' mapping approach but not during the 'APR-specific' mapping approach. SNP markers in bold were detected in both mapping approaches

${ }^{\mathrm{b}} \mathrm{Chr}$ ' 0 ' indicates unmapped SNPs that were significant in the analysis

${ }^{c} R^{2}$ Indicates percent of phenotypic variation explained by the significant locus

${ }^{d-g}$ The ' + ' sign indicates that the SNP was also detected in GWAS results in each of the environments. The '-' sign indicates that the SNP was detected only in combined analysis of all environments, and not in individual environments

Table 2 List of SNPS significantly associated with seedling resistance to Ug99 (race TTKSK)

\begin{tabular}{|c|c|c|c|c|}
\hline SNP & $\mathrm{Chr}^{\mathrm{a}}$ & Pos (cM) & $P$ value & $\mathrm{R}^{2}(\%)^{\mathrm{b}}$ \\
\hline IWB2159 & 0 & 0.0 & $3.15 \mathrm{E}-06$ & 4.8 \\
\hline IWB14222 & 0 & 0.0 & $3.45 \mathrm{E}-04$ & 2.8 \\
\hline IWB36324 & 0 & 0.0 & $6.44 \mathrm{E}-04$ & 2.5 \\
\hline IWB37066 & 0 & 0.0 & $3.62 \mathrm{E}-06$ & 4.8 \\
\hline IWB40312 & 0 & 0.0 & 1.97E-07 & 6.1 \\
\hline IWB49537 & 0 & 0.0 & $2.81 \mathrm{E}-04$ & 2.9 \\
\hline IWB68926 & 0 & 0.0 & 3.96E-05 & 3.7 \\
\hline IWA642 & $1 \mathrm{D}$ & 67.7 & $1.32 \mathrm{E}-04$ & 3.2 \\
\hline IWB24497 & $3 B$ & 67.5 & 8.43E-06 & 4.4 \\
\hline IWB30621 & $3 B$ & 67.5 & $1.97 \mathrm{E}-07$ & 6.1 \\
\hline IWB42046 & $3 B$ & 67.5 & 8.27E-08 & 6.5 \\
\hline IWB4823 & $3 B$ & 67.5 & 8.43E-06 & 4.4 \\
\hline IWB56471 & $3 B$ & 67.5 & $1.97 \mathrm{E}-07$ & 6.1 \\
\hline IWB61425 & $3 B$ & 67.5 & 8.43E-06 & 4.4 \\
\hline IWB59929 & $3 B$ & 74.4 & 3.91E-05 & 3.7 \\
\hline IWB9451 & $3 B$ & 76.9 & $3.36 \mathrm{E}-04$ & 2.8 \\
\hline IWA5363 & $4 \mathrm{~A}$ & 40.3 & 7.90E-04 & 2.4 \\
\hline IWA3394 & $5 B$ & 132.3 & $2.29 \mathrm{E}-05$ & 3.9 \\
\hline IWB7593 & $5 B$ & 132.3 & $6.14 \mathrm{E}-04$ & 2.5 \\
\hline IWB822 & $5 B$ & 134.1 & $5.30 \mathrm{E}-04$ & 2.6 \\
\hline IWB46318 & $5 B$ & 215.7 & $1.56 \mathrm{E}-05$ & 4.1 \\
\hline IWA2099 & $5 B$ & 216.7 & $9.72 \mathrm{E}-05$ & 3.3 \\
\hline IWA2100 & $5 B$ & 216.7 & 1.13E-04 & 3.3 \\
\hline
\end{tabular}

${ }^{\mathrm{a}} \mathrm{Chr}$ ' 0 ' indicates unmapped SNPs that were significant in the analysis

${ }^{b} R^{2}$ Indicates the percent of phenotypic variation explained by the significant locus 
Table 3 List of SNPS significantly associated with seedling resistance to race TRTTF

\begin{tabular}{|c|c|c|c|c|c|c|c|c|c|}
\hline & & & & & & & & & \\
\hline SNP & $\mathrm{Chr}^{\mathrm{a}}$ & Pos (cM) & $P$ value & $R^{2}(\%)^{b}$ & IWA7006 & $6 \mathrm{~A}$ & 12.8 & $2.06 \mathrm{E}-05$ & 24.3 \\
\hline IWB843 & 0 & 0.0 & $1.43 \mathrm{E}-04$ & 15.4 & IWB22036 & $6 \mathrm{~A}$ & 12.9 & $4.64 \mathrm{E}-20$ & 20.2 \\
\hline IWB8113 & 0 & 0.0 & 8.36E-04 & 13.2 & IWB10105 & $6 \mathrm{~A}$ & 13.5 & $5.80 \mathrm{E}-10$ & 16.4 \\
\hline IWB9699 & 0 & 0.0 & 8.36E-04 & 12.9 & IWB11315 & $6 \mathrm{~A}$ & 13.5 & 7.37E-18 & 15.4 \\
\hline IWB25143 & 0 & 0.0 & 4.62E-07 & 10.8 & IWB23521 & $6 \mathrm{~A}$ & 13.5 & $5.98 \mathrm{E}-13$ & 15.4 \\
\hline IWB27028 & 0 & 0.0 & 5.95E-04 & 10.7 & IWB26414 & $6 \mathrm{~A}$ & 13.5 & $6.11 \mathrm{E}-12$ & 15.4 \\
\hline IWB48121 & 0 & 0.0 & $1.83 \mathrm{E}-04$ & 7.7 & IWB35219 & $6 \mathrm{~A}$ & 13.5 & $1.18 \mathrm{E}-17$ & 15.4 \\
\hline IWB64530 & 0 & 0.0 & 8.36E-04 & 7.6 & IWB43805 & $6 \mathrm{~A}$ & 13.5 & $1.98 \mathrm{E}-18$ & 14.8 \\
\hline IWB67724 & 0 & 0.0 & $2.26 \mathrm{E}-04$ & 6.1 & IWB43810 & $6 \mathrm{~A}$ & 13.5 & $1.85 \mathrm{E}-05$ & 14.8 \\
\hline IWB68822 & 0 & 0.0 & 8.36E-04 & 4.2 & IWB58271 & $6 \mathrm{~A}$ & 13.5 & $2.78 \mathrm{E}-19$ & 14.8 \\
\hline IWB9794 & $1 \mathrm{~B}$ & 43.9 & $6.06 \mathrm{E}-04$ & 4.2 & IWB60233 & $6 \mathrm{~A}$ & 13.5 & $6.11 \mathrm{E}-12$ & 14.8 \\
\hline IWB72495 & 1B & 53.3 & $6.06 \mathrm{E}-04$ & 4.1 & IWB6358 & $6 \mathrm{~A}$ & 13.5 & $1.85 \mathrm{E}-05$ & 12.5 \\
\hline IWB11356 & 1B & 62.4 & 5.66E-04 & 4.0 & IWB66015 & $6 \mathrm{~A}$ & 13.5 & $6.11 \mathrm{E}-12$ & 12.5 \\
\hline IWB11357 & $1 \mathrm{~B}$ & 62.4 & 5.66E-04 & 3.4 & IWB67413 & $6 \mathrm{~A}$ & 13.5 & 7.37E-18 & 12.5 \\
\hline IWB60559 & 1B & 62.4 & $6.06 \mathrm{E}-04$ & 3.2 & IWB67415 & $6 \mathrm{~A}$ & 13.5 & 8.35E-18 & 12.4 \\
\hline IWB65404 & 1B & 62.4 & 4.44E-04 & 3.2 & IWB72957 & $6 \mathrm{~A}$ & 13.5 & $1.98 \mathrm{E}-18$ & 12.4 \\
\hline IWB11819 & 1B & 62.6 & $6.06 \mathrm{E}-04$ & 3.2 & IWA272 & $6 \mathrm{~A}$ & 15.7 & $1.50 \mathrm{E}-04$ & 12.4 \\
\hline IWB11820 & 1B & 62.6 & 4.44E-04 & 3.2 & IWB64917 & $6 \mathrm{~A}$ & 15.7 & $3.29 \mathrm{E}-04$ & 11.9 \\
\hline IWB34561 & 1B & 62.6 & $6.06 \mathrm{E}-04$ & 3.6 & IWB5029 & $6 \mathrm{~A}$ & 16.0 & $2.84 \mathrm{E}-15$ & 11.8 \\
\hline IWB47684 & 1B & 62.6 & 7.00E-04 & 3.6 & IWB35595 & $6 \mathrm{~A}$ & 16.6 & $2.57 \mathrm{E}-12$ & 10.0 \\
\hline IWB53143 & 1B & 62.6 & $9.38 \mathrm{E}-04$ & 3.5 & IWB43808 & $6 \mathrm{~A}$ & 16.6 & $2.57 \mathrm{E}-12$ & 9.1 \\
\hline IWB6592 & $1 \mathrm{~B}$ & 62.6 & $6.06 \mathrm{E}-04$ & 3.5 & IWB64918 & $6 \mathrm{~A}$ & 16.6 & $2.57 \mathrm{E}-12$ & 7.6 \\
\hline IWB41306 & 1B & 64.9 & $6.06 \mathrm{E}-04$ & 3.4 & IWB72956 & $6 \mathrm{~A}$ & 16.6 & $2.57 \mathrm{E}-12$ & 6.5 \\
\hline IWB44021 & $1 \mathrm{D}$ & 8.7 & $5.95 \mathrm{E}-04$ & 3.4 & IWA7913 & $6 \mathrm{~A}$ & 17.0 & $2.57 \mathrm{E}-12$ & 6.1 \\
\hline IWA8551 & $1 \mathrm{D}$ & 50.6 & 7.02E-04 & 3.4 & IWB23519 & $6 \mathrm{~A}$ & 17.0 & $5.44 \mathrm{E}-10$ & 6.0 \\
\hline IWB24961 & $5 D$ & 200.3 & 3.70E-04 & 3.4 & IWA705 & $6 \mathrm{~A}$ & 20.0 & 5.56E-06 & 6.0 \\
\hline IWB57210 & $5 \mathrm{D}$ & 200.3 & 3.70E-04 & 3.4 & IWA4962 & $6 \mathrm{~A}$ & 21.1 & 4.26E-07 & 5.4 \\
\hline IWB12448 & $6 \mathrm{~A}$ & 1.9 & 4.82E-04 & 3.4 & IWB31479 & $6 \mathrm{~A}$ & 21.1 & $4.72 \mathrm{E}-07$ & 5.4 \\
\hline IWB33595 & $6 \mathrm{~A}$ & 3.4 & $1.14 \mathrm{E}-04$ & 3.4 & IWB48751 & $6 \mathrm{~A}$ & 21.1 & $1.01 \mathrm{E}-08$ & 5.4 \\
\hline IWB11274 & $6 \mathrm{~A}$ & 4.7 & 3.93E-08 & 3.3 & IWB20143 & $6 \mathrm{~A}$ & 22.0 & $2.44 \mathrm{E}-10$ & 5.4 \\
\hline IWB53755 & $6 \mathrm{~A}$ & 4.7 & 1.25E-04 & 3.2 & IWA4551 & $6 \mathrm{~A}$ & 22.9 & $6.94 \mathrm{E}-11$ & 5.4 \\
\hline IWA5416 & $6 \mathrm{~A}$ & 5.6 & 2.81E-09 & 3.4 & IWA4552 & $6 \mathrm{~A}$ & 22.9 & $1.11 \mathrm{E}-10$ & 5.4 \\
\hline IWA5781 & $6 \mathrm{~A}$ & 5.7 & 3.17E-09 & 3.3 & IWB22191 & $6 \mathrm{~A}$ & 22.9 & $6.67 E-06$ & 5.4 \\
\hline IWB7601 & $6 \mathrm{~A}$ & 6.4 & $1.85 \mathrm{E}-05$ & 3.7 & IWB28421 & $6 \mathrm{~A}$ & 22.9 & $2.44 \mathrm{E}-10$ & 5.4 \\
\hline IWB47842 & $6 \mathrm{~A}$ & 7.0 & $1.31 \mathrm{E}-04$ & 3.7 & IWB50019 & $6 \mathrm{~A}$ & 22.9 & $2.44 \mathrm{E}-10$ & 5.3 \\
\hline IWA3856 & $6 \mathrm{~A}$ & 12.5 & $1.85 \mathrm{E}-05$ & 28.6 & IWB28338 & $6 \mathrm{~A}$ & 23.0 & $6.67 \mathrm{E}-06$ & 4.8 \\
\hline IWA6871 & $6 \mathrm{~A}$ & 12.5 & $1.85 \mathrm{E}-05$ & 27.2 & IWB1550 & $6 \mathrm{~A}$ & 25.5 & $2.19 \mathrm{E}-10$ & 4.4 \\
\hline IWB23520 & $6 \mathrm{~A}$ & 12.5 & $1.85 \mathrm{E}-05$ & 25.7 & IWB22216 & $6 \mathrm{~A}$ & 25.5 & $5.42 \mathrm{E}-05$ & 4.3 \\
\hline IWB2392 & $6 \mathrm{~A}$ & 12.5 & $6.41 \mathrm{E}-12$ & 25.7 & IWB30507 & $6 \mathrm{~A}$ & 25.5 & $2.19 \mathrm{E}-10$ & 4.3 \\
\hline IWB26415 & $6 \mathrm{~A}$ & 12.5 & $1.85 \mathrm{E}-05$ & 24.7 & IWB40111 & $6 \mathrm{~A}$ & 25.5 & 5.36E-06 & 3.8 \\
\hline IWB43804 & $6 \mathrm{~A}$ & 12.5 & 1.85E-05 & 24.7 & IWB52325 & $6 \mathrm{~A}$ & 25.5 & $2.06 \mathrm{E}-10$ & 3.5 \\
\hline
\end{tabular}

Table 3 List of SNPs significantly associated with seedling resistance to race TRTTF (Continued) 
Table 3 List of SNPs significantly associated with seedling resistance to race TRTTF (Continued)

\begin{tabular}{llrll}
\hline IWB64084.2 & 6B & 9.8 & $3.68 \mathrm{E}-05$ & 5.3 \\
IWB11653.2 & 6B & 14.5 & $2.10 \mathrm{E}-05$ & 5.0 \\
IWB14901 & 7A & 124.3 & $2.72 \mathrm{E}-04$ & 3.9 \\
IWB48466 & 7A & 217.0 & $8.24 \mathrm{E}-04$ & 3.2
\end{tabular}

${ }^{a} \mathrm{Chr}$ ' 0 ' indicates unmapped SNPs that were significant in the analysis

${ }^{\mathrm{b}} \mathrm{R}^{2}$ Indicates the percent of phenotypic variation explained by the significant locus 
Table 4 List of SNPs significantly associated with seedling resistance to race TKTTF

\begin{tabular}{|c|c|c|c|c|c|c|c|c|c|}
\hline SNP & $\mathrm{Chr}^{\mathrm{a}}$ & Pos (cM) & $P$ value & $R^{2}(\%)^{b}$ & IWB29568 & $4 \mathrm{~A}$ & 164.1 & $1.69 \mathrm{E}-07$ & 5.4 \\
\hline IWB31876 & 0 & 0.0 & $8.08 \mathrm{E}-04$ & 9.7 & IWB3001 & $4 \mathrm{~A}$ & 164.1 & $3.44 \mathrm{E}-07$ & 5.3 \\
\hline IWB71333 & 0 & 0.0 & 4.84E-04 & 9.6 & IWB31447 & $4 \mathrm{~A}$ & 164.1 & 5.51E-07 & 5.0 \\
\hline IWB57448 & $1 \mathrm{~A}$ & 21.5 & 8.57E-04 & 9.3 & IWB34609 & $4 \mathrm{~A}$ & 164.1 & 1.17E-06 & 4.9 \\
\hline IWA8622 & $1 \mathrm{~A}$ & 24.4 & $2.26 \mathrm{E}-04$ & 9.2 & IWB36388 & $4 \mathrm{~A}$ & 164.1 & 4.52E-07 & 4.9 \\
\hline IWB22324 & $4 \mathrm{~A}$ & 142.3 & $5.22 \mathrm{E}-06$ & 9.1 & IWB4517 & $4 \mathrm{~A}$ & 164.1 & $1.46 \mathrm{E}-05$ & 4.9 \\
\hline IWA4651 & $4 \mathrm{~A}$ & 144.4 & $3.10 \mathrm{E}-08$ & 8.8 & IWB48829 & $4 \mathrm{~A}$ & 164.1 & $9.10 \mathrm{E}-07$ & 4.9 \\
\hline IWB27971 & $4 \mathrm{~A}$ & 144.4 & 1.97E-05 & 7.5 & IWB49256 & $4 \mathrm{~A}$ & 164.1 & 5.08E-07 & 4.7 \\
\hline IWB34478 & $4 \mathrm{~A}$ & 144.4 & 3.37E-05 & 6.8 & IWB52393 & $4 \mathrm{~A}$ & 164.1 & $1.16 \mathrm{E}-06$ & 4.2 \\
\hline IWB34733 & $4 \mathrm{~A}$ & 144.4 & $1.54 \mathrm{E}-11$ & 4.9 & IWB6097 & $4 \mathrm{~A}$ & 164.1 & $2.58 \mathrm{E}-06$ & 3.9 \\
\hline IWB3569 & $4 \mathrm{~A}$ & 144.4 & 3.95E-05 & 3.9 & IWB72383 & $4 \mathrm{~A}$ & 164.1 & $2.60 \mathrm{E}-08$ & 3.7 \\
\hline IWB61312 & $4 \mathrm{~A}$ & 144.4 & 3.95E-05 & 3.7 & IWB9276 & $4 \mathrm{~A}$ & 164.1 & 1.90E-07 & 3.7 \\
\hline IWB63979 & $4 \mathrm{~A}$ & 144.4 & 1.19E-09 & 3.5 & IWA2224 & $5 \mathrm{~A}$ & 88.0 & 8.03E-04 & 3.5 \\
\hline IWB68386 & $4 \mathrm{~A}$ & 144.4 & $8.24 \mathrm{E}-04$ & 3.2 & IWA2836 & $5 \mathrm{~A}$ & 94.9 & 5.08E-04 & 3.2 \\
\hline IWA1505 & $4 \mathrm{~A}$ & 145.2 & 1.15E-08 & 3.1 & IWB34927 & $5 \mathrm{~A}$ & 94.9 & 9.63E-04 & 3.2 \\
\hline IWA3449 & $4 \mathrm{~A}$ & 147.1 & 3.83E-05 & 3.9 & IWB72540 & $6 \mathrm{~B}$ & 108.9 & $6.96 \mathrm{E}-04$ & 3.5 \\
\hline IWB2554 & $4 \mathrm{~A}$ & 147.1 & 1.61E-05 & 3.2 & IWA3268 & $6 \mathrm{~B}$ & 109.9 & 2.33E-04 & 3.2 \\
\hline IWB62397 & $4 \mathrm{~A}$ & 147.1 & 3.09E-04 & 13.9 & IWA5605 & $6 \mathrm{~B}$ & 109.9 & 3.19E-04 & 3.1 \\
\hline IWB12146 & $4 \mathrm{~A}$ & 150.7 & 5.47E-07 & 11.1 & IWA5606 & $6 \mathrm{~B}$ & 109.9 & 9.46E-04 & 10.1 \\
\hline IWB47019 & $4 \mathrm{~A}$ & 150.7 & 4.39E-07 & 9.2 & IWB56595 & $6 \mathrm{~B}$ & 109.9 & 2.40E-04 & 9.3 \\
\hline IWB59346 & $4 \mathrm{~A}$ & 150.7 & 5.93E-06 & 8.7 & IWB2749 & $6 \mathrm{~B}$ & 110.4 & $7.59 \mathrm{E}-04$ & 9.3 \\
\hline IWB1407 & $4 \mathrm{~A}$ & 151.2 & 5.19E-05 & 8.1 & IWB2751 & $6 \mathrm{~B}$ & 110.4 & 4.59E-04 & 8.3 \\
\hline IWB14910 & $4 \mathrm{~A}$ & 151.2 & 3.47E-05 & 8.0 & IWB43467 & $6 \mathrm{~B}$ & 110.4 & $5.88 \mathrm{E}-04$ & 7.4 \\
\hline IWB71978 & $4 \mathrm{~A}$ & 151.2 & 3.47E-05 & 8.0 & IWB48603 & $6 \mathrm{~B}$ & 110.4 & 4.34E-04 & 7.3 \\
\hline IWB59368 & $4 \mathrm{~A}$ & 151.3 & $3.14 \mathrm{E}-04$ & 7.8 & IWB50367 & $6 \mathrm{~B}$ & 110.4 & 1.37E-04 & 6.7 \\
\hline IWB68322 & $4 \mathrm{~A}$ & 151.3 & $9.43 \mathrm{E}-06$ & 7.6 & IWB56594 & $6 \mathrm{~B}$ & 110.4 & 5.88E-04 & 6.7 \\
\hline IWB53393 & $4 \mathrm{~A}$ & 153.0 & 1.35E-04 & 7.6 & IWB61565 & $6 \mathrm{~B}$ & 110.4 & $3.01 \mathrm{E}-04$ & 6.7 \\
\hline IWB35434 & $4 \mathrm{~A}$ & 154.1 & 8.01E-04 & 7.5 & IWB65679 & $6 \mathrm{~B}$ & 110.4 & $6.45 \mathrm{E}-04$ & 6.1 \\
\hline IWB51926 & $4 \mathrm{~A}$ & 154.3 & $2.22 \mathrm{E}-04$ & 7.5 & IWB43133 & $6 \mathrm{~B}$ & 113.3 & 5.16E-04 & 5.3 \\
\hline IWB59099 & $4 \mathrm{~A}$ & 154.3 & 9.09E-06 & 7.4 & IWB61528 & $6 \mathrm{~B}$ & 113.3 & $5.16 \mathrm{E}-04$ & 5.2 \\
\hline IWB70193 & $4 \mathrm{~A}$ & 154.3 & 5.34E-06 & 7.4 & IWB14375 & $6 \mathrm{~B}$ & 113.7 & $1.68 \mathrm{E}-04$ & 4.8 \\
\hline IWB35545 & $4 \mathrm{~A}$ & 163.8 & 9.37E-07 & 7.4 & IWB1747 & $6 \mathrm{~B}$ & 113.7 & 5.46E-04 & 4.7 \\
\hline IWA1410 & $4 \mathrm{~A}$ & 164.1 & 5.23E-08 & 7.1 & IWB30381 & $6 \mathrm{~B}$ & 113.7 & $7.14 \mathrm{E}-04$ & 4.4 \\
\hline IWA4084 & $4 \mathrm{~A}$ & 164.1 & 2.39E-08 & 7.0 & IWB41515 & $6 \mathrm{~B}$ & 113.7 & $4.78 \mathrm{E}-05$ & 4.2 \\
\hline IWA4858 & $4 \mathrm{~A}$ & 164.1 & 4.55E-07 & 6.9 & IWB57727 & $6 \mathrm{~B}$ & 113.7 & $7.14 \mathrm{E}-04$ & 4.2 \\
\hline IWA5353 & $4 \mathrm{~A}$ & 164.1 & $1.49 \mathrm{E}-06$ & 6.9 & IWB58200 & $6 \mathrm{~B}$ & 113.7 & $3.42 \mathrm{E}-04$ & 4.2 \\
\hline IWA7364 & $4 \mathrm{~A}$ & 164.1 & $2.46 \mathrm{E}-08$ & 6.5 & IWB59006 & $6 \mathrm{~B}$ & 113.7 & $1.88 \mathrm{E}-05$ & 4.2 \\
\hline IWA7365 & $4 \mathrm{~A}$ & 164.1 & 1.38E-08 & 6.4 & IWB59306 & $6 \mathrm{~B}$ & 113.7 & $6.29 \mathrm{E}-07$ & 4.1 \\
\hline IWB21715 & $4 \mathrm{~A}$ & 164.1 & $5.74 \mathrm{E}-08$ & 6.0 & IWB70316 & $6 \mathrm{~B}$ & 113.7 & $2.35 \mathrm{E}-04$ & 4.1 \\
\hline IWB23332 & $4 \mathrm{~A}$ & 164.1 & 2.85E-07 & 6.0 & IWB72471 & $6 \mathrm{~B}$ & 113.7 & $1.52 \mathrm{E}-04$ & 4.1 \\
\hline IWB26256 & $4 \mathrm{~A}$ & 164.1 & 2.37E-06 & 6.0 & IWB9416 & $6 \mathrm{~B}$ & 113.7 & $4.59 \mathrm{E}-06$ & 4.1 \\
\hline IWB26495 & $4 \mathrm{~A}$ & 164.1 & 1.85E-07 & 5.7 & IWA4245 & $6 \mathrm{~B}$ & 114.4 & $2.10 \mathrm{E}-04$ & 4.0 \\
\hline IWB275 & $4 \mathrm{~A}$ & 164.1 & 3.45E-07 & 5.7 & IWA4246 & $6 \mathrm{~B}$ & 116.2 & $8.75 \mathrm{E}-04$ & 3.9 \\
\hline IWB27679 & $4 \mathrm{~A}$ & 164.1 & 5.06E-04 & 5.4 & IWB28557 & $6 \mathrm{~B}$ & 116.2 & 4.91E-04 & 3.9 \\
\hline
\end{tabular}

Table 4 List of SNPs significantly associated with seedling resistance to race TKTTF (Continued)

0

9

9

7 
Table 4 List of SNPS significantly associated with seedling resistance to race TKTTF (Continued)

\begin{tabular}{|c|c|c|c|c|}
\hline IWB59175.2 & $6 \mathrm{~B}$ & 119.0 & 4.47E-04 & 3.9 \\
\hline IWB24880 & $6 \mathrm{~B}$ & 120.3 & $1.35 \mathrm{E}-04$ & 3.8 \\
\hline IWB24881 & $6 \mathrm{~B}$ & 120.3 & 8.13E-04 & 3.7 \\
\hline IWB41216 & $6 \mathrm{~B}$ & 120.3 & 7.51E-04 & 3.7 \\
\hline IWB24882 & $6 \mathrm{~B}$ & 120.6 & 1.35E-04 & 3.5 \\
\hline IWB3553 & $6 \mathrm{~B}$ & 120.6 & 5.79E-05 & 3.5 \\
\hline IWB45581 & $6 \mathrm{~B}$ & 120.6 & 2.20E-05 & 3.5 \\
\hline IWB46893 & $6 \mathrm{~B}$ & 120.6 & 9.30E-05 & 3.5 \\
\hline IWB66027 & $6 \mathrm{~B}$ & 120.6 & $5.14 \mathrm{E}-07$ & 3.4 \\
\hline IWB10711.2 & $6 \mathrm{~B}$ & 121.8 & $2.38 \mathrm{E}-08$ & 3.4 \\
\hline IWB23602 & $6 \mathrm{~B}$ & 121.8 & 1.39E-04 & 3.4 \\
\hline IWB23603 & $6 \mathrm{~B}$ & 121.8 & 1.86E-04 & 3.4 \\
\hline IWB40587 & $6 \mathrm{~B}$ & 121.8 & 1.69E-04 & 3.4 \\
\hline IWB44802 & $6 \mathrm{~B}$ & 121.8 & 1.57E-06 & 3.3 \\
\hline IWB73072 & $6 \mathrm{~B}$ & 121.8 & $2.25 \mathrm{E}-08$ & 3.3 \\
\hline IWB48548 & $6 \mathrm{~B}$ & 121.9 & $1.57 \mathrm{E}-06$ & 3.3 \\
\hline IWB28880 & $6 \mathrm{~B}$ & 122.1 & 1.57E-06 & 3.3 \\
\hline IWB44669 & $6 \mathrm{~B}$ & 122.1 & $1.08 \mathrm{E}-07$ & 3.3 \\
\hline IWB464 & $6 \mathrm{~B}$ & 122.1 & 7.18E-04 & 3.3 \\
\hline IWB71190 & $6 \mathrm{~B}$ & 122.2 & 2.71E-04 & 3.2 \\
\hline IWB43213 & $6 \mathrm{~B}$ & 122.3 & 1.69E-04 & 3.2 \\
\hline IWB41217 & $6 \mathrm{~B}$ & 122.9 & $6.96 \mathrm{E}-04$ & 3.2 \\
\hline IWB47075 & $6 \mathrm{~B}$ & 122.9 & $6.28 \mathrm{E}-09$ & 3.2 \\
\hline IWB34899.2 & $7 \mathrm{~A}$ & 6.4 & $3.22 \mathrm{E}-05$ & 5.0 \\
\hline
\end{tabular}

${ }^{a} \mathrm{Chr}$ ' 0 ' indicates unmapped SNPs that were significant in the analysis ${ }^{\mathrm{b}} \mathrm{R}^{2}$ Indicates the percent of phenotypic variation explained by the significant locus 
Table 5 Elite spring wheat lines from North American breeding programs that exhibit high level of adult plant resistance (APR) to Ug99 in four field environments

\begin{tabular}{|c|c|c|c|c|c|c|}
\hline \multirow[t]{2}{*}{$\operatorname{Line}^{a}$} & \multirow[t]{2}{*}{ Origin $^{b}$} & \multicolumn{4}{|c|}{ Environment ${ }^{c}$} & \multirow[t]{2}{*}{ Avg severity ${ }^{\mathrm{d}}$} \\
\hline & & KenOff13 & KenMain13 & EthOff13 & EthOff14 & \\
\hline Park & Alberta & 25 & 5 & 20 & 20 & 18 \\
\hline 9262 & CIMMYT & NA & 5 & 30 & 30 & 22 \\
\hline AC_Cadillac & Manitoba & 18 & 5 & 20 & 2 & 11 \\
\hline AC_Splendor & Manitoba & 8 & 5 & 11 & NA & 8 \\
\hline Glencross & Manitoba & 18 & 15 & 40 & 5 & 19 \\
\hline Peace & Manitoba & NA & 5 & 10 & 5 & 7 \\
\hline Fortuna & MSU & 8 & 5 & 6 & 10 & 7 \\
\hline Hi-Line & MSU & NA & 5 & 30 & 30 & 22 \\
\hline Newana & MSU & NA & 5 & 13 & 20 & 13 \\
\hline Thatcher & MSU & 10 & 5 & 10 & 20 & 11 \\
\hline AC_Eatonia & Saskatchewan & NA & 5 & 40 & 10 & 18 \\
\hline CDC_Alsak & Saskatchewan & 5 & 5 & 35 & 10 & 14 \\
\hline CDC_Osler & Saskatchewan & NA & 5 & 5 & 5 & 5 \\
\hline Neepawa & Saskatchewan & 8 & 5 & 35 & 10 & 14 \\
\hline Roblin & Saskatchewan & NA & 5 & 25 & NA & 15 \\
\hline Selkirk & Saskatchewan & 10 & 5 & 10 & 50 & 19 \\
\hline $10010-20$ & UCD & 15 & 5 & 20 & 10 & 13 \\
\hline UC1642 & UCD & NA & 5 & 45 & 20 & 23 \\
\hline UC1682 & UCD & 10 & 5 & 25 & 50 & 23 \\
\hline MN03119-4 & UMN & NA & 10 & 45 & 20 & 25 \\
\hline MN03148 & UMN & 25 & 5 & 25 & 20 & 19 \\
\hline MN08013-2 & UMN & 10 & 5 & 30 & 50 & 24 \\
\hline HW080169 & WSU & NA & 5 & 40 & 30 & 25 \\
\hline Avg_APR_Lines & - & 18 & 8 & 31 & 28 & 21 \\
\hline Avg_GWAS_Panel & - & 33 & 35 & 52 & 50 & 43 \\
\hline
\end{tabular}

a'Avg_APR_Lines' represents the mean disease severity (\%) across the lines showing high level of APR, and 'Avg_GWAS_Panel' represents the mean disease severity among all lines in the GWAS panel

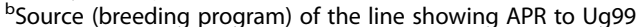

'Disease severity (\%) for each environment

${ }^{\mathrm{d}}$ The average disease severity (\%) across four environments 
Table 6 Elite spring wheat lines from North American breeding programs that exhibit high level of seedling resistance to race TTKSK. For each line, the observed seedling infection type (IT) for each race and the corresponding value on the linear scale are presented under the column 'IT' and 'Linear Score', respectively

\begin{tabular}{|c|c|c|c|c|c|c|c|}
\hline \multirow[t]{2}{*}{ Line $^{a}$} & \multirow[t]{2}{*}{ Origin $^{b}$} & \multicolumn{2}{|l|}{ TTKSK } & \multicolumn{2}{|l|}{ TRTTF } & \multicolumn{2}{|l|}{ TKTTF } \\
\hline & & $\mathrm{IT}$ & Linear Score & $\mathrm{IT}$ & Linear Score & $\mathrm{IT}$ & Linear Score \\
\hline 9253 & CIMMYT & $2-/ 3+$ & 4 & $2 / 3+$ & 5 & $2-$ & 4 \\
\hline 9262 & CIMMYT & 2 & 5 & 2 & 5 & ;2- & 1 \\
\hline 9263 & CIMMYT & ;12- & 1 & $; 1$ & 1 & $0 ; / 33+$ & 0 \\
\hline AC_Cadillac & Manitoba & $22+$ & 5 & 2 & 5 & $0 ; 1$ & 1 \\
\hline Peace & Manitoba & $22+$ & 5 & $2-$ & 3 & 0 & 0 \\
\hline Hi-Line & MSU & $0 ; 3-$ & 2 & $2-$ & 4 & 33- & 9 \\
\hline MT0415 & MSU & 2 & 5 & $33+$ & 8 & 01 & 1 \\
\hline Thatcher & MSU & $0 ; / 3+$ & 0 & $33+$ & 8 & $33+$ & 8 \\
\hline AC_Crystal & Saskatchewan & $2+$ & 6 & 2 & 5 & $32+$ & 7 \\
\hline AC_Karma & Saskatchewan & 2 & 5 & 2 & 5 & $22+$ & 5 \\
\hline AC_Vista & Saskatchewan & $22+$ & 5 & 2 & 5 & $22+$ & 5 \\
\hline SD4214 & SDSU & $2+$ & 6 & $3+$ & 9 & $12-$ & 3 \\
\hline SD4279 & SDSU & 2 & 5 & 2 & 5 & $33+$ & 9 \\
\hline PI610750 & UCD & $22+$ & 5 & $2-$ & 4 & $2-$ & 4 \\
\hline UC1643 & UCD & 2 & 5 & $22-$ & 5 & $0 ; 1$ & 1 \\
\hline Avg_Resistant_Lines & - & - & 4 & - & 5 & - & 4 \\
\hline Avg_GWAS_Panel & - & - & 8 & - & 7 & - & 4 \\
\hline
\end{tabular}

a'Avg_ Resistant_Lines' and 'Avg_GWAS_Panel' represent the mean linear score among lines resistant to TTKSK, and all lines in the GWAS panel, respectively

bource (breeding program) of the line showing APR to Ug99

\section{Author details}

'Department of Agronomy, Purdue University, 915 West State Street, West Lafayette 47907, IN, USA. ²Department of Agronomy and Plant Genetics, University of Minnesota, St. Paul 55108, MN, USA. ${ }^{3}$ United States Department of Agriculture-Agricultural Research Service (USDA-ARS), Cereal Disease Laboratory, St. Paul 55108, MN, USA. Department of Plant Pathology, University of Minnesota, St. Paul 55108, MN, USA. ${ }^{5}$ Department of Crop and Soil Sciences, Washington State University, Pullman 99164, WA, USA. ${ }^{6}$ International Maize and Wheat Improvement Center (CIMMYT), ICRAF House, United Nations Avenue, Gigiri, Nairobi, Kenya. ${ }^{7}$ United States Department of Agriculture-Agricultural Research Service (USDA-ARS), Aberdeen 83210, ID, USA. ${ }^{8}$ Kenya Agricultural and Livestock Research Organization (KALRO), Njoro, Kenya. ${ }^{9}$ Ethiopian Institute of Agricultural Research (EIAR), Pawe, Ethiopia.

Received: 11 December 2015 Accepted: 14 December 2015

Published online: 19 January 2016

\section{Reference}

1. Bajgain P, Rouse MN, Bulli P, Bhavani S, Gordon T, Wanyera R, et al. Association mapping of North American spring wheat breeding germplasm reveals loci conferring resistance to Ug99 and other African stem rust races. BMC Plant Biol. 2015;15:249.

Submit your next manuscript to BioMed Central and we will help you at every step:

- We accept pre-submission inquiries

- Our selector tool helps you to find the most relevant journal

- We provide round the clock customer support

- Convenient online submission

- Thorough peer review

- Inclusion in PubMed and all major indexing services

- Maximum visibility for your research

Submit your manuscript at www.biomedcentral.com/submit
Biomed Central 